\title{
The Information Bandwidth of Neutron Scattering Instruments
}

M W Johnson

July 1995 


\section{(C) Council for the Central Laboratory of the Research Councils 1995}

Enquiries about copyright, reproduction and requests for additional copies of this report should be addressed to:

The Central Laboratory for the Research Councils

Library and Information Services

Rutherford Appleton Laboratory

Chilton

Didcot

Oxfordshire

OX11 OQX

Tel: 01235445384 Fax: 01235446403

E-mail library@rl.ac.uk

ISSN 1358-6254

Neither the Council nor the Laboratory accept any responsibility for loss or damage arising from the use of information contained in any of their reports or in any communication about their tests or investigations. 


\title{
The Information Bandwidth of Neutron Scattering Instruments
}

\author{
M W Johnson \\ Rutherford Appleton Laboratory, \\ Chilton, Didcot, Oxon OQ11 0QX, UK
}

\begin{abstract}
The concept of the information content of a scientific measurement is introduced, and a theory is presented which enables the information that may be obtained by a neutron scattering instrument to be calculated. When combined with the time taken to perform the measurement the bandwidth of the instrument is obtained. This bandwidth is effectively a figure of merit which is of use in three respects: in the design of neutron instrumentation, the optimisation of measurements, and in the comparison of one instrument with another.
\end{abstract}

\section{Introduction}

The purpose of this paper is to offer a new tool for assessing the 'usefulness' of instruments. We will do this by quantifying the rate at which information may be obtained using a neutron diffractometer or spectrometer. The techniques used may be straightforwardly extended to any other counting instruments.

What is information? A theory of information transmission was first developed by Shannon [1] and its application to science has been explored by Bruillouin [2], and Jaynes [3], among others. This theory disentangles the qualitative aspects of information content (which are nonetheless important ) from the quantitative aspects. In this paper we apply this theory to instrumental measurements.

Consider a system $S$ which, as far as an observer is concerned, can be in $\Lambda_{0}$ equally probable states. If further information is given to the observer, which reduces the number states that $S$ can be in to $\Lambda_{1}$, then the content $(I)$ of this information is defined as:

$$
I=K \ln \left(\Lambda_{0} / \Lambda_{1}\right)
$$

This definition of information results in an additive rule for independent information. Thus if information is received in two stages, our knowledge about a system changing from $\Lambda_{0}$ to $\Lambda_{1}$ to $\Lambda_{2}$, the total information received is equal to the sum of the information received:

$$
I_{T}=K \ln \left[\frac{\Lambda_{0}}{\Lambda_{2}}\right]=K \ln \left[\frac{\Lambda_{0}}{\Lambda_{1}} \frac{\Lambda_{1}}{\Lambda_{2}}\right]=K \ln \left[\frac{\Lambda_{0}}{\Lambda_{1}}\right]+K \ln \left[\frac{\Lambda_{1}}{\Lambda_{2}}\right]=I_{1}+I_{2}
$$

In a similar way a message may be said to contain information $I_{M}$ :

$$
I_{M}=K \ln \left(M_{0} / M_{1}\right)
$$


where $M_{0}$ and $M_{1}$ are the number of possible messages before and after the message was received. If the message is unambiguous then $M_{l}$ will equal unity and the information content of the message simply equal to $K \ln \left(M_{0}\right)$. Thus if a signalling system can transmit a binary digit of length $n$ bits, the number of possible messages will be $2^{n}$, and the information content will be given by:

$$
I_{M}=K \ln \left(2^{n}\right)
$$

The coefficient $K$ in the above expressions is arbitrary, but if chosen to be equal to $1 / \ln (2)$ then the information is expressed in units of 'bits'. Thus substituting this value for $K$ into equation 4 gives a value for $I_{M}$ of $\mathrm{n}$ bits, in agreement with the binary message length.

In a similar way, the use of an instrument to perform a measurement may be considered a transmission of information. We may calculate the quantity of information in a similar way, by considering the number of outcomes that were possible before the measurement, and compare it to the number possible after the measurement.

\section{The scalar counter}

All neutron scattering instruments are essentially counting devices. We must therefore begin by establishing the information contained in an experiment involving a single scalar counter. The number of possibilities before the measurement is clearly related to the maximum count that can be measured by the scalar in the time chosen. With a maximum count of $M$, it could be argued that the information contained in the measurement is:

$$
I=K \ln (M+1)
$$

since one possible count has been determined $\left(\Lambda_{1}\right)$ from the $M+1$ (including zero) possible before the measurement occurred $\left(\Lambda_{0}\right)$. This is too superficial since it ignores the effect of statistical noise. The number of possibilities after the measurement is related to the probability distribution of the 'true count' $\theta$ that may be derived from the actual count $k$. When this is taken into account the result, which is derived in section D of the appendix, is:

$$
I=K \ln \sqrt{M / 2 \pi}
$$

Having obtained the information content of a single scalar counter it is necessary to consider the effects of backgrounds. Such backgrounds may be broadly of two sorts, those that are independent of the actual count, $k$, but may be regarded as a constant fraction of the maximum possible count $(b=f . M)$, and those that are linearly related to the actual count $k(b=f . k)$. Of course the background could be a mixture of these two types, or related in a more complex way to both.

Here we will consider the first type of background. Using the result (C4 - appendix) we may, quite generally write the information content of a pair of counts $(k, 1)$ from foreground and background measurements: 


$$
I_{\text {sample }}=K \sum_{k} \sum_{l} p_{k l} \ln \left(\Lambda_{0} / \Lambda_{k l}\right)
$$

where $p_{k l}$ is the probability of the pair of measurements, and $\Lambda_{0}, \Lambda_{k l}$ the possibilities before and after the measurements. The details of the calculation are given in the appendix (section E) and give the result:

$$
I_{s}=K \ln \sqrt{\frac{M}{2 \pi}}+K \ln \left[\frac{(1-f)}{(1+f)^{1 / 2}}\left(\frac{2 f}{1+f}\right)^{f /(1-f)}\right]
$$

where the background $b=f M$.

The equation (8) may be written

$$
I_{s}=K \ln \sqrt{\frac{c M}{2 \pi}}
$$

where the maximum count $M$ is now modified by the factor $\mathrm{c}$, which is given by

$$
c=\left[\frac{(1-f)}{(1+f)^{1 / 2}}\left(\frac{2 f}{1+f}\right)^{f /(1-f)}\right]^{2}
$$

Thus $c(f)$ is a measure of the effective reduction in the maximum count $M$ due to the presence of a background, $b=f M$. A plot of $c(f)$ is given in Fig.1. It will be seen that $10 \%$ background equivalent to a $50 \%$ reduction in intensity, and $50 \%$ background equivalent to reducing the intensity by more than $90 \%$.

We may also use equation (9) to answer the question; " by what factor (g) must the intensity be increased to compensate for a background fraction of (f) ?". The result is approximated by $1 / \mathrm{c}$, but is derived exactly by the solution of the transcendental equation outlined in section $\mathrm{F}$ of the appendix. The result is shown graphically in Figure 2. This shows a similar result, where a background of $\sim 20 \%$ requires the signal intensity to be doubled to mitigate its effect, and a background of $\sim 75 \%$ requires the signal to be increased by a factor of 10 .

\section{A spectrum of scalar counts}

We are now in a position to evaluate the information content of measurement on an instrument that consists of a series of scalar counters. From the additive rule for independent measurements (2) the information contained in a spectrum of $\mathrm{n}_{\mathrm{c}}$ independent scalar counters will be : 


$$
I=\frac{K}{2} \ln \prod_{i=1, n_{\mathrm{c}}} \frac{c_{i} M_{i}}{2 \pi}
$$

where $c_{i}$ and $M_{i}$ are the background correction and maximum count for the $i^{\text {th }}$ scalar. If we can make the simplifying assumption that these are the same for all scalars then equation (11) reduces to:

$$
I_{s}=K n_{c} \ln \sqrt{\frac{c M}{2 \pi}}
$$

where $n_{c}$ is the number of channels in the spectrum of scalar counters.

Care must be taken in calculating $\mathrm{n}_{c}$, since it is the number of independent channels in a spectrum. Thus, in a time-of-flight spectrum with $n_{t}$ time channels $n_{c}$ is generally less than $n_{t}$ since the data recorded in adjacent time channels is not independent. To calculate the effective number of independent time channels we may use the equation ( B4 ) to determine the effective width $\Delta$ of a peak in a spectrum.

It is interesting to note that the effective width $\left(\Delta_{\mathrm{e}}\right)$ of an exponential peak with a shape $y=\exp \{-t / \tau\}$ is $\Delta_{e}=\tau$ which, when expressed in terms of the standard deviation $\sigma_{e}$ or full-width at half height $\left(w_{\mathrm{e}}\right)$ is $\Delta_{e}=\sigma_{e}=w_{e} / \ln 2=1.44 w$. For a Gaussian $\Delta_{g}=\sigma \sqrt{2 \pi e}$, and hence $\Delta_{g}=4.13 \sigma_{g}=1.76 w_{g}$. Thus it will be seen that a Gaussian has an effective width that is greater than an exponential peak of similar half-width or of similar standard deviation. In fact it has been shown [4] that, for a given standard deviation, the Gaussian is the widest (least informative) peak shape of all. The values for $\Delta$, FWHMH and $\sigma$ for four common peak shapes are given in Table 1 .

Knowing the effective peak width in a time spectrum $\left(\Delta_{t}\right)$ we may calculate the number of independent time channels by dividing the time axis into channels with these widths. For example a time-of-flight pattern in which the exponential peak shape varies such that $w=r t$ (where $r=5.10^{-4}$ ) the number of independent time channels is given by

$$
n_{t}=\int_{t_{1}}^{t_{2}} \frac{d t}{\Delta_{t}}=\int_{t_{1}}^{t_{2}} \frac{d t}{1.44 r t}=\frac{1}{1.44 r} \ln \left(t_{2} / t_{1}\right)
$$

which for the HRPD instrument at ISIS operating between 1 and $5 \AA$ gives a value for $n_{t}$ of 2235. It is also straightforward to show that, if instead of choosing to describe the powder diffraction pattern as a time spectrum, we had worked in units of neutron momentum ( $Q \propto 1 / t)$, the number of momentum channels $n_{Q}$ is the same. We may therefore rewrite equation (13) in terms of the momentum channels $\mathrm{n}_{\mathrm{q}}$, or , for an inelastic spectrometer in terms of the energy channels $n_{e}$, so long as each is calculated using the effective width parameter in the appropriate units, and is calculated from the resolution function using equation (B4).

For instruments which measure both momentum and energy changes, for which the resolution in energy and momentum are independent, and in which all channels have equal $\mathrm{c}$ and $\mathrm{M}$ : 


$$
I=K n_{Q} n_{E} \ln \sqrt{\frac{c M}{2 \pi}}
$$

In situation where $\Delta_{q}$ and $\Delta_{w}$ are not independent, the product $n_{q} n_{w}$ must be replaced by $n_{\mathcal{C}}$ channels, representing the number of independent areas into which the $q-w$ plane may be divided, using a $2-\mathrm{d}$ version of equation (B4).

\section{Different sample states}

By altering the conditions of the sample, changing its temperature for example, we are given new information about a, potentially, different sample. An instrument that is capable of changing sample temperature from 200 to $1000 \mathrm{C}$ in steps of $2 \mathrm{C}$ can provide 400 independent measurements. Thus we may combine the results outlined above to arrive at the complete equation for the information that may be obtained from a single sample:

$$
I=K n_{s} n_{c} \ln \sqrt{\frac{c M}{2 \pi}}
$$

The above equation combines our knowledge about the count-rate, background, resolution and range of the instrument together with the sample environment ranges of an instrument to produce a figure for the information content of a measurement or series of measurements on a single sample.

\section{The bandwidth of an instrument}

So far we have left out of this analysis the time taken to do experiments. Clearly an instrument that takes a long time to set up and make a measurement is inferior to one that is quick to operate. For this reason we wish to express the total effectiveness of an instrument as a bandwidth - and calculate the rate at which instruments can acquire information. Introducing the term $\mathrm{R}$ for the maximum rate that the $\mathrm{i}^{\text {th }}$ channel can count, gives for a single sample state:

$$
I=K n_{c} \ln \sqrt{\frac{c R\left(t-t_{s}\right)}{2 \pi}}
$$

Where $t_{\mathrm{s}}$ is the set-up time of the experiment.

To understand the variation of this information measure as a function of time it is shown in Figure 3. For a certain length of time the information gained is zero ( $\mathrm{O}$ to $\mathrm{A}$ in Figure 3 ). 
This time is comprised two parts: the set-up time and the time taken for the expected maximum effective count (i.e. $c R\left(t-t_{s}\right)$ ) to exceed $2 \pi$ ( since a scalar that has a maximum count below this value is providing no new information). From point $A$ onwards the information content increases - but only logarithmically.

The bandwidth (B) of the instrument may be defined by the rate, in ' bits / sec ', that information is obtained. The expression for this quantity is therefore given by:

$$
B=\frac{K n_{s} n_{c}}{t} \ln \sqrt{\frac{c R\left(t-t_{s}\right)}{2 \pi}}
$$

This measure is not constant and varies with the length of the measurement,t. It reaches a maximum at point $\mathbf{B}$ in Figure 3 and then declines. For later times, at $\mathbf{C}$ for example, the information gained will be greater, but the rate at which it has been acquired will be slower. It is interesting to note that the time at which the bandwidth is at its highest is when (if $c \approx 1.0$ )

$$
R t=2 \pi e
$$

which means after there are approximately 17 counts in the highest scalar. This is clearly at a point in the data collection at which there is too little information for the run to be complete but it does provide some explanation for the 'first neutrons are best' phenomenon. They certainly provide the most information per neutron of the whole run.

If we now consider a set of runs, each lasting a time $t_{r}$ the total information gained over a time $t$ will be:

$$
I=\frac{K n_{c} t}{t_{r}} \ln \sqrt{\frac{c R\left(t_{r}-t_{s}\right)}{2 \pi}}
$$

Such an equation is relevant for a series of different sample environment settings on the same sample, or in the case of a 3-AXIS instrument where data is recorded at a series of different orientations of the same sample. The above equation now allows us to compare the rate at which information can be collected, taking into account, intensity, backgrounds, resolution, temperature ranges and set up time.

To compare instruments with one another is known to be difficult, and since their bandwidths vary with time it is not possible to simply place instruments on a single real axis for comparison. It must also be remembered that in setting the values for $R$ and $n_{c}$ in equation 19 the values may be calculated for an ideal sample, to give the highest bandwidth possible for that instrument; or may be modified to account for the effects of measuring samples which will lower these ideal values. The instrument and sample must often be considered as a system if useful results are to be obtained. 
One way to proceed is to list the information that may be gathered, in certain lengths of time from a single, ideal sample. A simple instrument, a low resolution liquids diffractometer for example, will acquire information rapidly, but never achieve a very high value in reasonable length of time due to the logarithmic nature of the information gain. A very complex instrument, such as a triple-axis spectrometer, may have a lower data collection rate, but a much higher eventual information gain since the information is dominated in this case by the number of crystal settings that may be employed (thus affecting $\mathrm{n}_{\mathrm{c}}$ ) and not the count rate of the instrument.

Thus a useful way to proceed is to enumerate the information gain, from a single sample, that may be obtained from an instrument over certain lengths of time. In Table 2 this has been done for a range of time periods, and for a set of typical instruments. The results are also shown graphically in Fig. 4.

\section{Discussion}

The information content of a measurement, and the bandwidth of an instrument may provide measures of their usefulness, but how do these measures relate to the quality or quantity of 'science' that might be done using them? At the highest level the purpose of the measurement might be to provide evidence for the mechanism of high temperature superconductivity - but the connection between such high-level goals and the raw data is not straightforward.

What we have done, in determining the information content of a measurement made using a particular instrument, is to determine the discriminating power of the instrument. Thus we have effectively determined the total number of 'distinguishable data states' that a particular measurement can identify. We can call this the total number of states in d-space. However, raw data is not the object of interest. The end product of all data analysis is a parameterised model of the system under study. We may call this the p-space.

In crystallography, for example, the p-space consists of lists of atom types, positions and thermal amplitudes. In spectroscopic studies the p-space might consists of modes, frequencies and amplitudes. What does our knowledge of the maximum number of distinguishable d-states $\left(n_{d}\right)$ tell us about the number of distinguishable p-states $\left(n_{p}\right)$ ? The answer is that :

$$
n_{p} \leq n_{d}
$$

since in the mapping from $d$-space to $\mathrm{p}$-space:

i) one-to-many mappings are not allowed since they imply a single data state could give rise to more than one distinguishable $\mathrm{p}$-space state, and ii) many-to-one mappings reduce $\mathrm{n}_{\mathrm{p}}$.

The information content of the p-space data $\left(I_{p}\right)$ is related to that in the d-space $\left(I_{d}\right)$ through a similar relationship

$$
I_{p} \leq I_{d}
$$


This result derives from equation (21) above together with the fact that the probability of each of the d-space states was considered equally probable. Introducing non-equal probabilities into either $n_{d}$ or $n_{p}$ reduces the information content. It is interesting to compare the information content of a measurement on the High Resolution Powder Diffractometer (HRPD) at ISIS with one of its results in p-space. Table 3 shows the results of a single measurement on a powdered sample of benzene. In this table we can identify a total of 27 parameters described using approximately 120 decimal digits. The length of this p-space message is therefore around 400 bits, significantly less than the d-space information content of approximately 20 kbits.

\section{Acknowledgements}

My thanks to Bill David, Devinder Sivia, and Andrew Taylor who commented on the paper, and to Ruth Johnson who has helped to clarify many tortuous sentences.

\section{References}

1. C E Shannon \& W Weaver, The Mathematical Theory of Communication, (University of Illinois Press, Urbana \& Chicago, 1949), Illini Books ed. 1963.

2. L Brillouin, Science and Information Theory, (Academic Press, New York, 1962 )

3. E T Jaynes, Papers on Probability, Statistics and Statistical Physics, (D Reidel Publishing Co., Dordrecht, 1983) Ed. R D Rosenkrantz, Synthese Library, vol. 158.

4. A A Sveshnikov, Problems in Probability Theory, Mathematical Statistics and Theory of Random Functions, (Dover Publications, New York, 1978) 


\section{Appendix : Probabilities, Possibilities and Information Theory}

The definition of information proposed by Bruillouin (see equation 1), is cast in terms of two quantities, $\Lambda_{0}$ and $\Lambda_{1}$, the numbers of equally probable states before and after the receipt of the information. This expression therefore requires

and ii) the states to be equally probable.

The results derived in this paper require the calculation of $\Lambda_{0}$ and $\Lambda_{1}$ with three further generalisations:

a) where the states $\left\{s_{i}\right\}$ comprising $\Lambda$ are not equally probable,

b) where the states $\left(\mathrm{s}_{\mathrm{i}}\right\}$ comprising $\Lambda$ are continuous and not discrete,

c) where the outcome $\Lambda_{1}$ is not a single set of states $\left\{s_{i}\right\}$, but is a set of set of states $\left\{\left\{s_{i}\right\}_{1},\left\{s_{i}\right\}_{2},\left\{s_{i}\right\}_{3} \ldots \ldots,\left\{s_{i}\right\}_{n}\right\}$ each set $\left\{s_{i}\right\}_{j}$ having a probability $\mathrm{p}_{j}$.

These derivations are given in sections $\mathrm{a}, \mathrm{b}$ and $\mathrm{c}$ of this appendix. To assist in the clarity of the main text two further derivations are included in this appendix. In section (d) we calculate the information content of a scalar counter, and in section (e) the information content of a scalar counter including the effect of background.

\section{A) Possibilities of a Discrete Probability Distribution.}

In calculating results in information theory it is frequently required to calculate the 'number of possible ways' that a certain system might be in. For brevity we shall refer to the 'number of possible ways' as the possibility of the system and give it the symbol $\Lambda$.

We begin with a simple example. A message of $\mathrm{N}$ letters, drawn from an alphabet of $\mathbf{n}$ letters each with a probability $p_{i}$ of occurring, has a possibility $\Lambda\left(p_{i}, N\right)$ given (for large $N$ ) by:

$$
\Lambda=\frac{N !}{N_{1} ! N_{2} ! \ldots N_{n} !}
$$

where $N_{i}=p_{i} N$. Using Stirling approximation gives the result:

$$
\Lambda=\exp \left\{-N \sum p_{i} \ln p_{i}\right\}
$$

The expression occurring in the exponential term in A2

$$
S=-\sum p_{i} \ln p_{i}
$$

is the usual expression for the entropy of a probability distribution first established by Shannon [1].

To get some feel for the meaning of $S$ consider the case of an alphabet of $\mathrm{n}$ equally probable letters. In this case 


$$
S=-\sum \frac{1}{n} \ln \left(\frac{1}{n}\right)
$$

hence

$$
n=e^{s}
$$

Thus, in the case of an alphabet with letters of equal probability, $\exp (S)$ is exactly the same as the number of letters in the alphabet. Where the probabilities are not uniform $\exp (S)$ provides a useful measure of the equivalent number of equally probable letters $\left(n^{\prime}\right)$ such that if $\Lambda$ is calculated for a message of $\mathrm{N}$ letters from the formula

$$
\Lambda=\left(n^{\prime}\right)^{N}=(\exp (S))^{N}=\exp (N S)
$$

the correct result (i.e. A2) is obtained.

Consider now the following problem. Our prior knowledge about a system is that it may be in any one of $n$ states, each with a probability $\mathrm{p}_{\mathrm{i}}$. Following an experiment our knowledge of the system is modified so that the states now have probabilities pi'. The question is; 'what is the information content of this change from $p_{i}$ to $p_{i^{\prime}}$ ?' To do this we need to count the number of states before and after the experiment. Simply counting the number of different states, $\mathrm{n}$, clearly overstates the total number since a very small number of the states might represent $99 \%$ of the total probability. We require a measure that satisfies two criteria:

a) if there are $n$ states of equal probability the measure should equal $n$

b) if each state is divided into $q$ with probabilities $\mathrm{p} / \mathrm{q}$ the total number of states should increase by a factor of $q$.

We have already seen that if we use the measure

$$
\Lambda=\exp \left\{-\sum p_{i} \ln p_{i}\right\}
$$

this satisfies the first of these requirements.

Dividing each state into $q$ sub-states will result in a POSSIBILITY of:

$$
\begin{aligned}
& \Lambda^{\prime}=\exp \left\{-q \sum\left(\frac{p_{i}}{q}\right) \ln \left(\frac{p_{i}}{q}\right)\right\} \\
& =\exp \left\{-\sum p_{i} \ln \frac{p_{i}}{q}\right\} \\
& =\exp \left\{-\sum p_{i} \ln p_{i}-\sum p_{i} \ln q\right\} \\
& \therefore \Lambda^{\prime}=q \exp \left\{-\sum p_{i \mathrm{n}} p_{i}\right\}=q \Lambda
\end{aligned}
$$


which is the desired result.

We can therefore use the expression A7 as the measure of the number of states in a system when not all are equally probable.

\section{B) Possibilities of a Continuous Probability Distribution Function.}

In section A above we have seen that the number of states of a discrete probability distribution function (pdf) is given by expression A7. What is the corresponding expression for a continuous pdf, and what is its meaning? For a discrete pdf $\Lambda$ is simply a measure of the number of states, and if all are equally probable, is equal to the total number. We would therefore expect $\Lambda$ for the continuous case to represent a length, and in the case of a rectangular distribution to be equal to its width.

We can turn the continuous case into a discrete case by dividing the continuous $p(x)$ up into lengths $\delta \mathrm{x}$. Thus $\Lambda$ now becomes:

$$
\begin{aligned}
\Lambda & =\exp \left\{-\sum\left(\delta x p\left(x_{i}\right)\right) \ln \left(\delta x p\left(x_{i}\right)\right)\right\} \\
& =\exp \left\{-\sum \delta x p\left(x_{i}\right) \ln \delta x-\sum \delta x p\left(x_{i}\right) \ln p\left(x_{i}\right)\right\} \\
& =\frac{1}{\delta x} \exp \left\{-\sum \delta x p\left(x_{i}\right) \ln p\left(x_{i}\right)\right\}
\end{aligned}
$$

The exponential term in $\mathrm{B} 3$ has the dimensions of length and when divided by the term $\delta \mathrm{x}$ results in the (dimensionless) number $\Lambda$. Thus $\delta$ x plays the role of a scaling constant, depending on the resolution with which we divide $p(x)$. We may therefore write the effective width $(\Delta)$ of the function $p(x)$ as:

$$
\Delta=\exp \left\{-\int d x p(x) \ln p(x)\right\}
$$

noting that the quantity $\Delta$ has the dimensions of length. To check that B4 gives the expected answer for a rectangular distribution

substitution in B4 gives:

$$
\begin{array}{ll}
p(x)=1 / a & 0 \leq x \leq a \\
p(x)=0 & \text { otherwise }
\end{array}
$$

$$
\Delta=\exp \left\{-\int_{0}^{a} d x(1 / a) \ln (1 / a)\right\}=a
$$

the desired result. 


\section{Section C : The Information Content involving multiple sets of states}

If after a measurement, instead of a final state with $\Lambda_{1}$ possibilities, there are $\mathrm{n}$ possible final states each with differing possibilities $\Lambda_{1}, \Lambda_{2}, \Lambda_{3}, \ldots . \Lambda_{n}$, what is the information content? Let the final states have probabilities $\mathrm{p}_{1}, \mathrm{p}_{2}, \ldots \mathrm{p}_{\mathrm{n}}$, and the initial possibility of the system be $\Lambda_{0}$. Consider an ensemble of $\aleph$ measurements. This will result in $\kappa p_{1}, \kappa p_{2}, \ldots \kappa p_{n}$ final states each with possibilities $\Lambda_{1}, \Lambda_{2}, \Lambda_{3}, \ldots . \Lambda_{n}$.

If we now measure the information content of the entire ensemble of $*$ measurements, the total number of starting possibilities is $\Lambda_{0}^{\kappa}$ and the total number of final possibilities is $\Lambda_{1}^{p_{1} \kappa} \Lambda_{2}^{p_{2} \kappa} \ldots . \Lambda_{n}^{p_{n} \kappa}$

The information for the ensemble of measurements is therefore:

$$
\begin{aligned}
& I=K \ln \left(\Lambda_{0}^{\aleph} / \prod_{i=1}^{n} \Lambda_{i}^{p_{i} \aleph}\right) \\
& I=K \ln \prod_{i=1}^{n}\left(\frac{\Lambda_{0}^{p_{i} \aleph}}{\Lambda_{i}^{p_{i} \aleph}}\right) \\
& I=K \aleph \sum_{i=1}^{n} p_{i} \ln \left(\Lambda_{0} / \Lambda_{i}\right)
\end{aligned}
$$

But this is the information content for an ensemble of $\aleph$ measurements, and the average information content of a single experiment is therefore

$$
I=K \sum_{i=1}^{n} p_{i} \ln \left(\Lambda_{0} / \Lambda_{i}\right)
$$

\section{Section D The Information content of a Scalar Counter}

We may calculate the probability of the true count being $\theta$ given the observed count, $\mathrm{k}$ using Bayes' theorem:

$$
P(\theta \mid k)=\frac{P(k \mid \theta) P(\theta)}{P(k)}
$$

with the result

$$
P(\theta \mid k)=\frac{e^{-\theta} \theta^{k}}{k !}
$$


Substituting this probability distribution function into B4 yields its effective width:

$$
\Delta=\sqrt{2 \pi e k}
$$

For continuous variables the information gain may be simply calculated using the ration of lengths, rather than possibilities, hence :

$$
I=K \ln \left(\Delta_{0} / \Delta_{1}\right)=K \ln ((M+1) / \sqrt{2 \pi e k})
$$

where $\Delta_{0}$ and $\Delta_{1}$ are the possible lengths $\theta$ may occupy before and after the measurement.

Since we wish to calculate the expected information gain from a scalar counter that might return any value between 0 and $M$, we need to know how to average the information gain when an experiment may have $\mathrm{n}$ outcomes with probability $p_{1}, p_{2}, \ldots \ldots p_{n}$ each with possibilities $\Lambda_{1}, \Lambda_{2} \ldots \ldots \Lambda_{n}$. The result, derived in section $C$ of the appendix, is:

$$
I=K \sum_{1}^{n} p_{i} \ln \left(\Lambda_{0} / \Lambda_{i}\right)
$$

For a scalar counter that may give values between 0 and M, equation D5 may be re-written as:

$$
I=K \sum_{k=0}^{M} p(k) \ln \left(\Delta_{0} / \Delta_{k}\right)
$$

Assuming no additional knowledge about the probability $\mathrm{p}(\mathrm{k})$ of the counter producing a result $\mathrm{k}$ we may set $p(k)=1 /(M+1)$, and use the result at D3 for $\Delta_{1}$, giving:

$$
I=K \frac{1}{M+1} \ln \left(\frac{M+1}{e}\right)+K \sum_{k=1}^{M} \frac{1}{(M+1)} \ln \left(\frac{M+1}{\sqrt{2 \pi e k}}\right)
$$

The first term in D6 being that for $\mathrm{k}=0$ for which $\Delta_{k}=e$.

Evaluating D6 gives the result for the average information obtained from a scalar counter with a maximum value of $\mathrm{M}$ :

$$
I=K\left(1+\frac{1}{2(M+1)}\right) \ln (M / 2 \pi)^{1 / 2}+K \frac{\ln 2 \pi}{2(M+1)}+K \frac{1}{M(M+1)}
$$

which is well approximated (for large $M$ ) by:

$$
I=K \ln \left(\frac{M}{2 \pi}\right)^{1 / 2}
$$




\section{Section E: The Information content of a Scalar Counter with Background}

We may treat the probabilities of particular foreground (k) or background (l) counts as independent (thus $p_{k l}=p_{k} p_{l}$ ). The important effect of the background is to restrict $\mathrm{k}$ to lie approximately between $b$ and $M$, to restrict 1 to lie near to $b$, and to increase the error in measuring the sample count to $\sigma^{2}$ sample $=\sigma^{2}{ }_{k}+\sigma^{2}{ }_{l}$. These conditions are well approximated by the following:

$$
\begin{aligned}
& p_{l}=1 \quad(l=b) \\
& p_{l}=0 \quad(\text { otherwise }) \\
& p_{k}=1 /(M-b) \quad(b \leq k \leq M) \\
& p_{k}=0 \quad \text { (otherwise) }
\end{aligned}
$$

and

$$
\begin{aligned}
& \Lambda_{0}=M-b \\
& \Lambda_{k l}=\sqrt{2 \pi e(k+b)}
\end{aligned}
$$

the values of the possibilities in (E2) reflecting the a priori knowledge that the count $\mathrm{k}$ lies between $\mathrm{b}$ and $\mathrm{M}$, and that the error on the sample measurement is $(k+b)$. Substituting $(\mathrm{E} 1, \mathrm{E} 2)$ in $\mathrm{D} 6$ gives

$$
I_{s}=K \ln \sqrt{\frac{M}{2 \pi}}+K \ln \left[\frac{(1-f)}{(1+f)^{1 / 2}}\left(\frac{2 f}{1+f}\right)^{f /(1-f)}\right]
$$

where $f=b / M$.

Section F: The increased signal required to compensate for the presence of background

The information content of a scalar count that has had its signal increased by a factor $\mathrm{g}$ is given by:

$$
I=\frac{K}{2} \ln \frac{(g M(1-f)+f M)}{2 \pi}+\frac{K}{2} \ln c^{\prime}
$$

where $c^{\prime}$ is given by the usual definition (equation 10 ) :

$$
c^{\prime}=\left[\frac{\left(1-f^{\prime}\right)}{\left(1+f^{\prime}\right)^{1 / 2}}\left(\frac{2 f^{\prime}}{1+f^{\prime}}\right)^{f^{\prime}\left(1-f^{\prime}\right)}\right]^{2}
$$


and $\mathrm{f}^{\prime}$ is given by :

$$
f^{\prime}=\frac{B}{g S+B}=\frac{f M}{g(1-f) M+f M}=\frac{f}{f+g(1-f)}
$$

Substituting $\mathrm{F} 3$ and $\mathrm{F} 2$ into $\mathrm{F} 1$ and equating $\mathrm{F} 1$ to $\frac{K}{2} \ln \left(\frac{M}{2 \pi}\right)$ provides the necessary condition for calculating the gain factor ' $\mathrm{g}$ ', which may then be calculated numerically. 


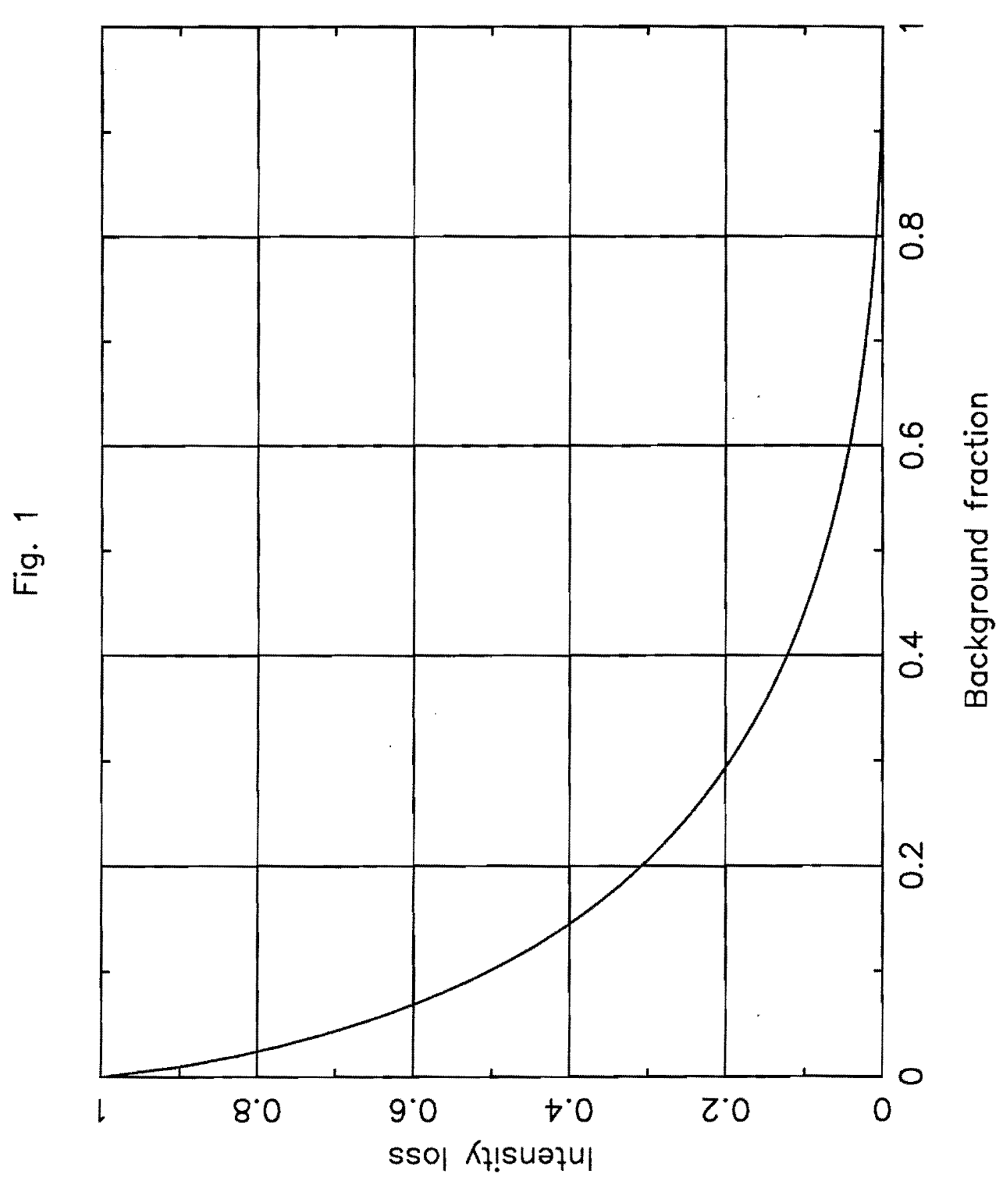




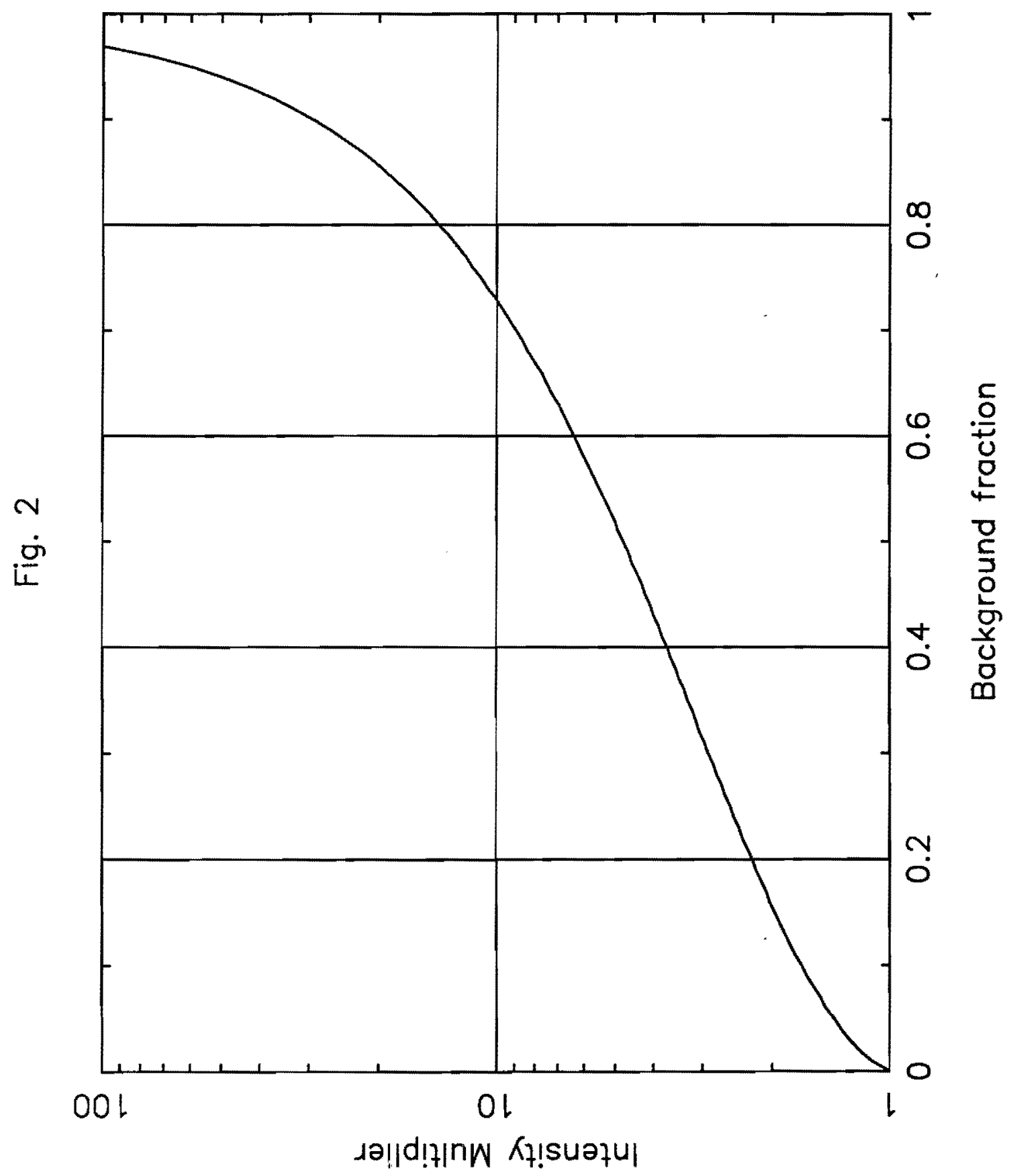




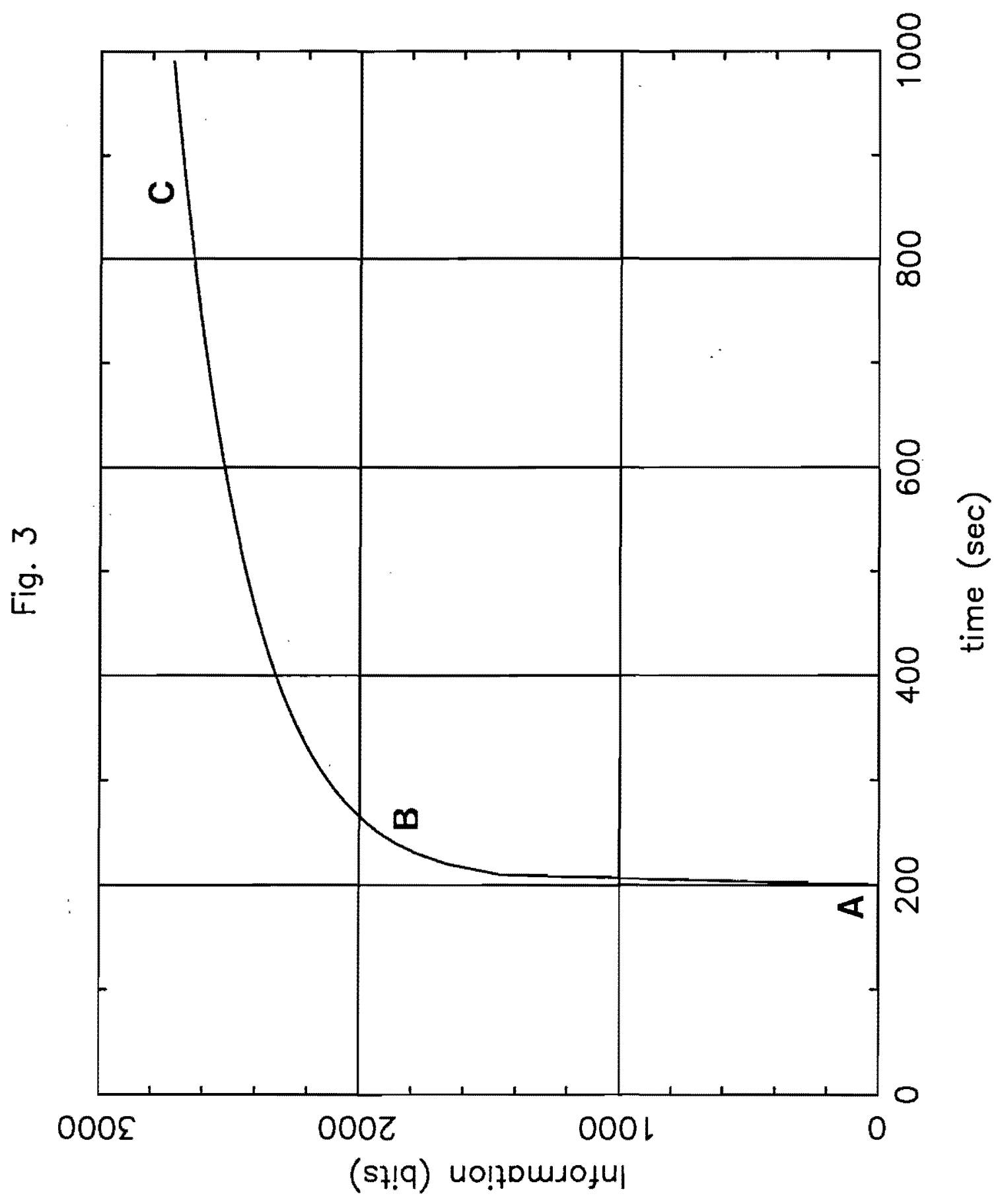




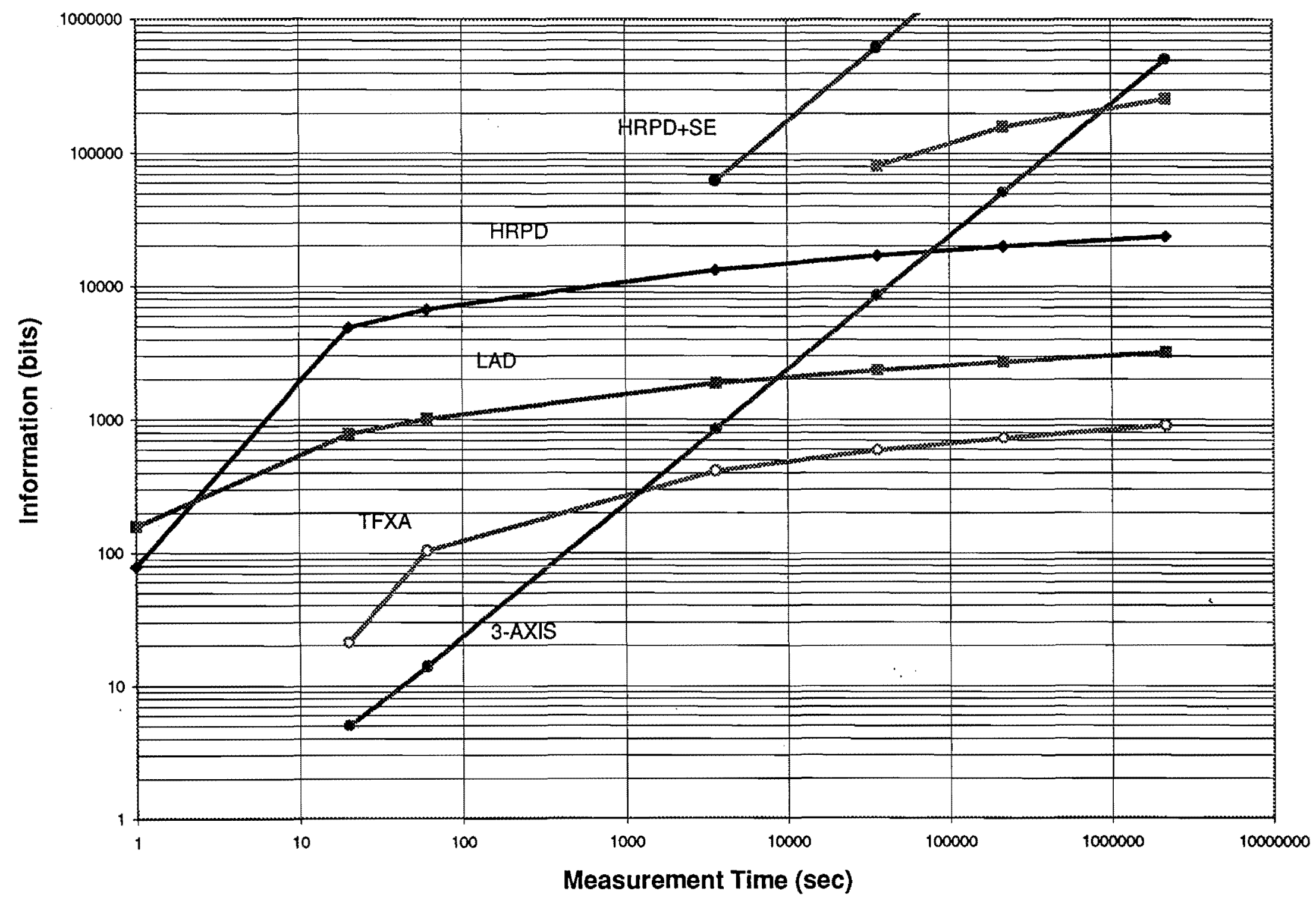

Fig. 4 


\begin{tabular}{|c|c|c|c|c|c|}
\hline A & Gaussian & $f=\exp \left\{-x^{2} / 2 \sigma^{2}\right\}$ & $\sigma \sqrt{2 \pi e}$ & $\sigma \sqrt{8 \ln 2}$ & $\sigma$ \\
\hline $\mathbf{D}$ & square & $f=1 / a$ & $\mathrm{a}$ & $\mathrm{a}$ & $a / \sqrt{12}$ \\
\hline $\mathbf{A}$ & triangular & $f=\frac{1}{a} \pm \frac{x}{a^{2}}$ & $a \sqrt{e}$ & $\mathrm{a}$ & $a / \sqrt{6}$ \\
\hline $\mathbf{\Lambda}$ & exponential & $f=\exp \{-x / a\}$ & $\mathrm{a}$ & $\mathrm{a} \ln 2$ & $\mathrm{a}$ \\
\hline
\end{tabular}

Table 1 
Single Sample

Information Gain

\begin{tabular}{|c|c|c|c|c|c|c|c|c|c|c|c|c|c|}
\hline instrument & $\mathrm{nc}$ & $R$ & $f$ & tr & ts & C & $1 \mathrm{sec}$ & 20 sec & $1 \mathrm{~min}$ & 1 hour & 10 hours & 2.5 days & 25 days \\
\hline HRPD & 2235 & 8 & 0.02 & & 0 & 0.82 & 79 & 4914 & 6686 & 13293 & 17009 & 19900 & 23616 \\
\hline HRPD + OC & 2235 & 8 & 0.02 & 500 & 300 & 0.82 & 0 & 0 & 0 & 62130 & 621297 & 3727780 & \\
\hline TFXA & 105 & 0.5 & 0.02 & & 0 & 0.82 & 0 & 21 & 104 & 414 & 589 & 725 & 899 \\
\hline MARI & 60000 & 0.0017 & 0.05 & & 0 & 0.67 & 0 & 0 & 0 & 0 & 80558 & 158177 & 257925 \\
\hline LAD & 288 & 20 & 0.05 & & 0 & 0.67 & 158 & 781 & 1009 & 1861 & 2339 & 2712 & 3191 \\
\hline
\end{tabular}

Table 2 
Benzene : cell dimensions and atomic positions from HRPD neutron diffraction at ISIS

\begin{tabular}{|l|l|l|l|l|}
\hline $\mathrm{a}=7.3551(3)$ & $\mathrm{b}=9.3712(4)$ & $\mathrm{c}=6.6994(3)$ & & \\
\hline
\end{tabular}

\begin{tabular}{|l|l|l|l|l|}
\hline c1 & $-0.06120(15)$ & $0.14123(10)$ & $-0.00519(20)$ & $68(6)$ \\
\hline c2 & $-0.14023(15)$ & $0.04469(10)$ & $0.12722(15)$ & $66(6)$ \\
\hline c3 & $0.07770(15)$ & $-0.09689(12)$ & $0.13264(20)$ & $77(6)$ \\
\hline d1 & $-0.10853(15)$ & $0.25050(15)$ & $-0.01187(25)$ & $202(9)$ \\
\hline d2 & $-0.24908(20)$ & $0.07682(15)$ & $0.22600(20)$ & $202(9)$ \\
\hline d3 & $-0.13821(20)$ & $-0.17136(15)$ & $0.23703(20)$ & $203(9)$ \\
\hline
\end{tabular}

Table 3 